\title{
Sox9-dependent expression of Gstm6 in Sertoli cells during testis development in mice
}

\author{
Annemiek Beverdam, Terje Svingen, Stefan Bagheri-Fam ${ }^{1}$, Pascal Bernard ${ }^{1}$, Peter McClive ${ }^{2}$, \\ Mathew Robson, Mahdi Banan Khojasteh, Mahboubeh Salehi, Andrew H Sinclair ${ }^{2}$, Vincent \\ R Harley ${ }^{1}$ and Peter Koopman
}

Institute for Molecular Bioscience, The University of Queensland, Brisbane, Queensland 4072, Australia, ${ }^{1}$ Human Molecular Genetics Laboratory, Prince Henry's Institute of Medical Research, Clayton, Victoria 3168, Australia and ${ }^{2}$ Department of Paediatrics, Murdoch Children's Research Institute, Royal Children's Hospital, The University of Melbourne, Melbourne, Victoria 3052, Australia

Correspondence should be addressed to P Koopman; Email: p.koopman@imb.uq.edu.au

A Beverdam and T Svingen contributed equally to this work

\begin{abstract}
Glutathione $S$-transferases (GSTs) are an important family of multifunctional enzymes that play a role in the protection of tissues by the detoxification of hazardous and carcinogenic compounds. We found previously that Gstm6 is upregulated in the somatic cells of male mouse fetal gonads relative to female gonads. In this study, we describe the spatial and temporal expression pattern of Gstm6 during mouse development. We show that Gstm6 is predominantly expressed in the reproductive system, at significantly higher levels in XY gonads compared with XX gonads from $11.5 \mathrm{dpc}$ onwards, and remains expressed in the testes in adult mice. Its expression is associated with the Sertoli cell lineage, and is dependent on the expression of the male sex-determining gene Sox9. Our data suggest that Gstm6 plays a male-specific role in gonad development or function, possibly by modulating the exposure of somatic tissue and/or germ cells to endogenous or exogenous toxicants.

Reproduction (2009) 137 481-486
\end{abstract}

\section{Introduction}

Sex determination is achieved in three stages during development. First, genetic sex is established by inheritance of one of both sex chromosomes, $\mathrm{X}$ or $\mathrm{Y}$, from the father. If the $\mathrm{Y}$-chromosome is present, the male sex-determining gene Sry is activated in pre-Sertoli cells residing in the bipotential gonads, immediately followed by the activation of a second male-determining gene Sox9, resulting in the development of testes (Koopman et al. 1990, 1991, Kent et al. 1996). In the absence of Sry, such as in female embryos, or in the absence of Sox 9 function (Barrionuevo et al. 2006), the gonads develop into ovaries. Later in fetal life and during puberty, the testes and ovaries produce male and female sex hormones respectively, which are important for the development of the reproductive system and secondary sex characteristics.

Sex determination and gonad development are orchestrated by intricate genetic programming, the nature of which we are only beginning to understand. To obtain insight into these processes, diverse microarray screens have been carried out and have provided a large number of candidate genes (Nef et al. 2005, Small et al. 2005, Beverdam \& Koopman 2006, Bouma et al. 2007, Clement et al. 2007, Cory et al. 2007). Many of these genes are either unknown or have not been implicated in the genetic network underlying sex determination and gonad development. The major challenge of the next decade is to understand how these genes take part in the genetic networks controlling sex determination and gonad development.

Glutathione $S$-transferases (GSTs) are an important family of multifunctional isoenzymes. They catalyse the conjugation of reduced glutathione via the sulphhydryl group, to electrophilic centres on a wide variety of substrates (Douglas 1987). This activity is useful in the detoxification of endogenous compounds such as peroxidised lipids as well as the metabolism of xenobiotics (reviewed by Hayes \& Pulford 1995, Hayes et al. 2005). In addition to their enzymatic activities, GSTs may also bind toxins and function as transport proteins.

Previously, we showed in a somatic gonad cell-typespecific microarray screen that glutathione S-transferase mu 6 (Gstm6) is highly upregulated (39-fold) in 11.5 days 
post-coitum (dpc) pre-Sertoli cells when compared with $10.5 \mathrm{dpc}$ pre-Sertoli cells and $11.5 \mathrm{dpc}$ pre-granulosa cells, the female equivalent of Sertoli cells (Albrecht \& Eicher 2001, Beverdam \& Koopman 2006). Here, we describe in-depth the spatial and temporal expression pattern of Gstm6 in the developing embryos and adult mouse tissues. We found that Gstm6 is expressed at high levels in the gonads and at lower levels in other embryonic mouse tissues. Gstm6 expression was initially restricted to testicular cords in $X Y$ gonads, but from around $14.5 \mathrm{dpc}$ onwards Gstm6 mRNA was also detected in the developing ovaries. In addition, we found that Gstm6 expression is restricted to the Sertoli cell lineage, and analysis of Sox9-knockout gonads indicated that its expression was dependent on Sox9. These data suggest that Gstm6 may have yet undiscovered roles in the earliest steps of male sex determination and/or gonad development.

\section{Results}

\section{Gstm6 expression in mouse embryos and adult gonads}

We first analysed the expression pattern of Gstm6 in whole $13.5 \mathrm{dpc}$ mouse embryos by section in situ hybridisation (SISH), and found robust expression of Gstm6 in the developing testes, and low or background expression in other fetal tissues (Fig. 1A). These results were confirmed by quantitative, real-time RT-PCR (qRTPCR) assays of a range of tissue samples from $13.5 \mathrm{dpc}$ embryos (Fig. 1B). These analyses reveal that Gstm6 is expressed at high levels in the developing testes compared with the developing ovaries and other fetal tissues. Furthermore, qRT-PCR of adult mouse gonadal tissue revealed high levels of expression in testes but not ovaries (Fig. 1C), indicating that testis-specific expression of Gstm6 continued into adult life.

\section{Temporal and cellular profile of Gstm6 expression}

We next analysed the temporal Gstm6 expression profiles by qRT-PCR in embryonic XY and XX gonads at stages just prior to, during and after the onset of male sex determination, which occurs around $11.5 \mathrm{dpc}$ (Fig. 2A). We found that at 10.5 and $11.5 \mathrm{dpc}$, the expression levels of Gstm6 were low both in XY and XX genital ridges. After $11.5 \mathrm{dpc}$, Gstm6 expression levels increased sharply in $\mathrm{XY}$ gonads to $16.5 \mathrm{dpc}$, the last stage examined. Curiously, expression levels were lower at $13.5 \mathrm{dpc}$ than at 12.5 or $14.5-16.5 \mathrm{dpc}$. This temporary decrease in expression levels was found consistently in several independent experiments and was also observed in in situ hybridisation experiments (see below). In XX gonads, expression levels remained low throughout the period tested, $10.5-16.5 \mathrm{dpc}$ (Fig. 2A).

To analyse the spatial expression pattern of Gstm6 in the developing gonads, we carried out in situ hybridisation assays on whole $\mathrm{XY}$ and $\mathrm{XX}$ gonads from 11.5 to $16.5 \mathrm{dpc}$ (Fig. 2B). Using this technique, we detected Gstm6 expression in $\mathrm{XY}$ but not in $\mathrm{XX}$ gonads at $11.5 \mathrm{dpc}$. From $12.5 \mathrm{dpc}$ onwards, Gstm6 staining was detected with increasing intensity in the developing testis cords, with lower levels of staining at $13.5 \mathrm{dpc}$, consistent with qRT-PCR data. In XX gonads, however, Gstm6 mRNA signal was not visible until $14.5 \mathrm{dpc}$, from which stage it was observed at low levels throughout the developing ovary (Fig. 2B).

To determine the cell type(s) expressing Gstm6 in XY gonads, we first carried out whole-mount in situ hybridisation (WISH) assays at $13.5 \mathrm{dpc}$ on wild-type and $W^{e} / W^{e}$ mutant testes that lack germ cells (Buehr et al. 1993). Gstm6 expression was detected in testis cords in $W / W^{e}$ samples at levels similar to wild-type $\mathrm{XY}$ gonads, suggesting that Gstm6 is expressed in Sertoli cells and/or peritubular myoid cells, and is independent of the presence of germ cells (Fig. 3A).

We then performed SISH assays on sagittally sectioned $X Y$ wild-type embryos at $13.5 \mathrm{dpc}$, and we found that Gstm6 was expressed within the testis cords in a pattern very similar to that of the Sertoli cell-specific marker anti-mullerian hormone $(A m h)$. In both cases, staining was observed in the irregularly shaped cytoplasms of Sertoli cells, and was stronger towards the periphery of
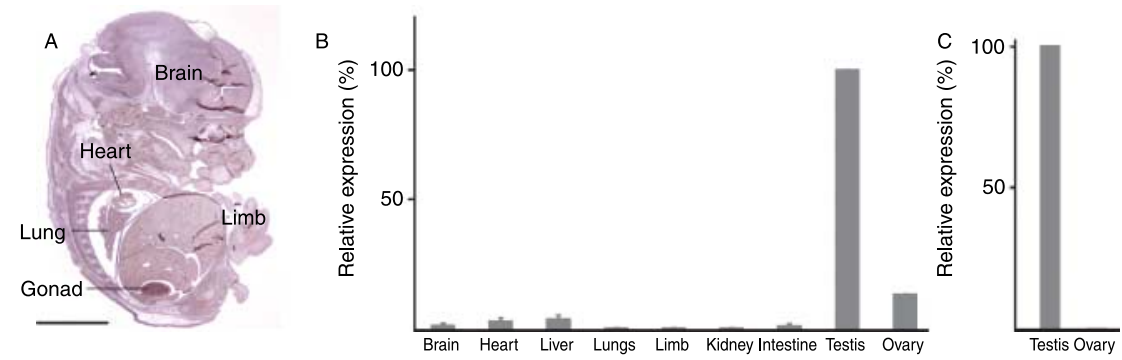

Figure 1 Gstm6 is specifically expressed in mouse embryonic and adult testes. (A) In situ hybridisation using Gstm6 probe 1 on a sagittal section of a $13.5 \mathrm{dpc}$ XY mouse embryo showing high levels of Gstm6 expression in the male gonad, whereas other embryonic tissues show little or no Gstm6 expression. Scale bar, $2 \mathrm{~mm}$. (B) qRT-PCR assays showing high levels of Gstm6 expression in 13.5 dpc testes and low levels in 13.5 dpc ovaries and other embryonic tissues. (C) qRT-PCR assays showing high levels of Gstm6 expression in adult testes, and low expression levels in adult ovaries. The Y-axes display relative fold expression levels compared with $18 S$ rRNA with the maximum expression level set to 100\%. Experiments were carried out three times and each experiment consisted of three replicates. The error bars represent the S.E.M. of three experiments. 

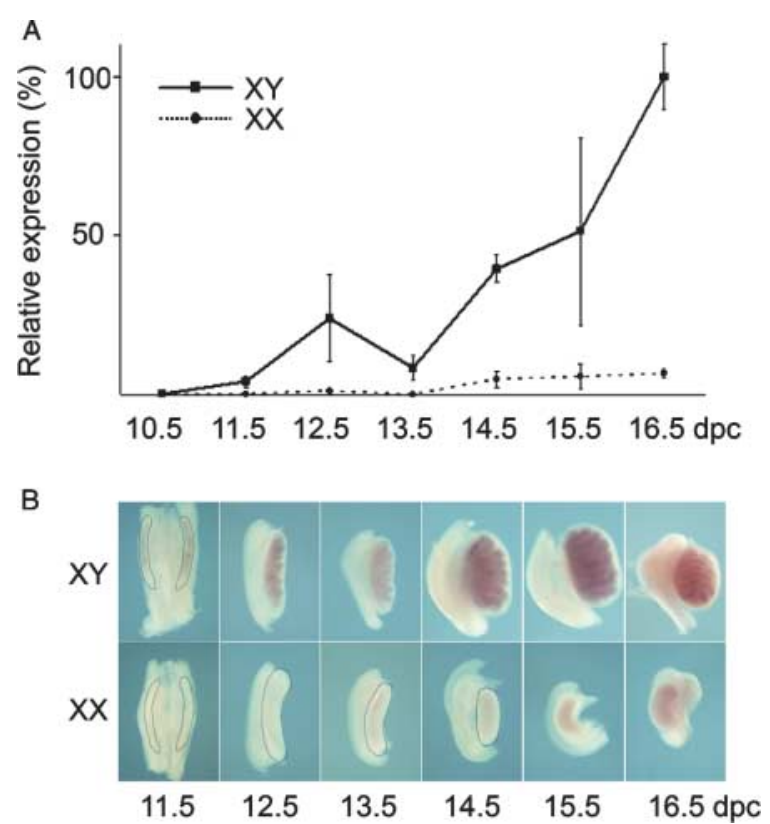

Figure 2 Gstm6 expression is upregulated in embryonic testes after sex determination. (A) qRT-PCR assays showing Gstm6 mRNA levels in XX and $\mathrm{XY}$ genital ridges and gonads from $10.5 \mathrm{dpc}$ to $16.5 \mathrm{dpc}$. Gstm6 expression is low in both $\mathrm{XY}$ and $\mathrm{XX}$ genital ridges at $10.5 \mathrm{dpc}$, but from 11.5 dpc Gstm6 expression is high in XY gonads, whereas it stays low in XX gonads. From $14.5 \mathrm{dpc}$, slightly higher levels of Gstm6 expression are observed also in XX gonads. The $Y$-axis displays relative fold expression levels compared with $18 S$ rRNA with the maximum expression level of each experiment set to $100 \%$. Experiments were carried out three times and each experiment consisted of three replicates. The error bars represent the S.E.M. of three experiments. (B) In situ hybridisation assays using Gstm6 probe 2 on whole XY (upper panels) and XX (lower panels) genital ridges and gonads from $11.5 \mathrm{dpc}$ to $14.5 \mathrm{dpc}$. Gstm6 is first activated in $11.5 \mathrm{dpc}$ XY embryos throughout the genital ridge, whereas no Gstm6 mRNA was detected in $11.5 \mathrm{dpc}$ $\mathrm{XX}$ genital ridges. From $12.5 \mathrm{dpc}$ onwards, Gstm6 expression is restricted to the testicular cords. At $14.5 \mathrm{dpc}$, Gstm6 expression was detected throughout the developing ovaries.

the cords (Fig. 3B). This staining was distinct from that obtained with probes detecting the germ cell marker Pou5f1, which was localised in the smooth, annular cytoplasms surrounding the nuclei of germ cells, and was stronger towards the centre of the cords (Fig. 3B). In addition, Gstm6 expression was also detected in a thin layer of cells surrounding the testis cords likely to be peritubular myoid cells (Fig. 3B, arrow).

qRT-PCR assays using RNA extracted from $13.5 \mathrm{dpc}$ wild-type and $W^{\mathrm{e}} / W^{\mathrm{e}}$ gonads corroborated our in situ hybridisation data (Fig. 3C-E). We analysed the expression levels of Gstm6 in comparison with the Sertoli cell-specific gene Sox 9 and the germ cell marker $D d x 4$ (Mvh, mouse vasa homologue; Toyooka et al. 2000). We found high levels of $D d x 4$ expression in wildtype gonads, but not $W^{e} / W^{e}$ gonads, confirming the absence of germ cells in the $W^{e} / W^{e}$ gonads (Fig. 3C). Sox9 expression levels were similar in wild-type and
$W^{e} / W^{e}$ gonads, as expected from a Sertoli cell-specific gene (Fig. 3D). Similarly, Gstm6 expression was detected in both wild-type and $W^{e} / W^{e}$ gonads at similar levels (Fig. 3E).

Our previous microarray analyses suggested that Gstm1 and Gstm2 are also expressed in a sexually dimorphic manner in the somatic cells of the developing gonads, albeit at lower levels than Gstm6 (Beverdam \& Koopman 2006). To further characterise the expression pattern of these two genes in the developing gonads, we performed qRT-PCR on wild-type and $W^{e} / W^{e}$ mutant $13.5 \mathrm{dpc}$ testes and ovaries. We found that the expression levels of Gstm1 and Gstm2 were higher in $13.5 \mathrm{dpc}$ XY WT gonads compared with $13.5 \mathrm{dpc}$ XX WT gonads (Fig. 3F and G). In addition, Gstm 1 and 2 were expressed at even higher levels in the absence of germ cells (Fig. 3F and G), suggesting that they are exclusively expressed by somatic gonad cells and not by germ cells, corroborating our microarray data.

\section{Gstm6 expression is dependent on Sox9}

Sox9 is known to regulate testis-specific expression of a number of genes, including Amh, Vanin 1, Pgds2 and Sf1 (Shen et al. 1994, De Santa Barbara et al. 1998, Wilson et al. 2005, Wilhelm et al. 2007). We therefore set out to determine whether Gstm6 expression was also dependent on the expression of Sox9, using a conditional knockout mouse model, in which Sox9 was inactivated in the developing gonads using the CRE/loxP recombination system, with CRE recombinase expressed under control of the cytokeratin 19 promoter (Barrionuevo et al. 2006). We determined the expression levels of Sox9 and Gstm6 by qRT-PCR in XY genital ridges and gonads from Sox9 conditional homozygous mutant embryos at $11.5,12.0$ and $12.5 \mathrm{dpc}$, relative to wildtype $X Y$ and $X X$ littermates. Sox9 expression levels in wild-type $X Y$ genital ridges and gonads increased from 11.5 to $12.5 \mathrm{dpc}$ and remained low in wild-type XX genital ridges and gonads, whereas Sox9 levels were strongly reduced in mutant genital ridges and gonads as expected (Fig. 4A). Gstm6 expression levels in wild-type XY genital ridges and gonads increased strongly between 11.5 and $12.5 \mathrm{dpc}$, but remained low in Sox9 mutant genital ridges and gonads (Fig. 4B). In fact, Gstm6 expression levels in Sox9 mutant genital ridges and gonads were similar to those found in wild-type $X X$ genital ridges and gonads. These results demonstrate that Gstm6 expression in XY genital ridges and gonads depends, directly or indirectly, on the expression of Sox9. The lag between upregulation of Sox 9 and Gstm6 in wild-type testes (Fig. 4), and the observation that both genes are expressed in Sertoli cells, further supports the hypothesis that Gstm6 acts downstream of Sox9 in the cascade of testis development. 
A

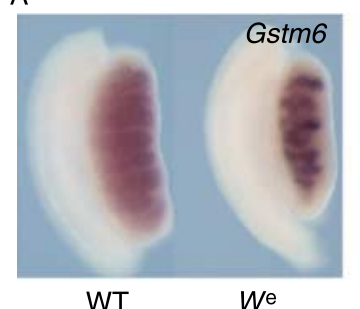

WT
We
B

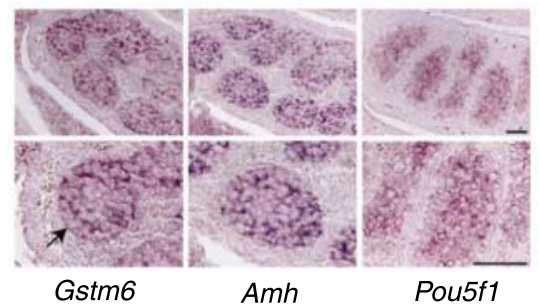

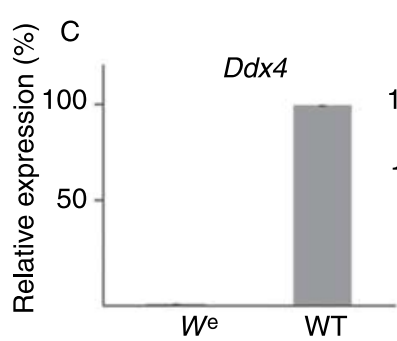

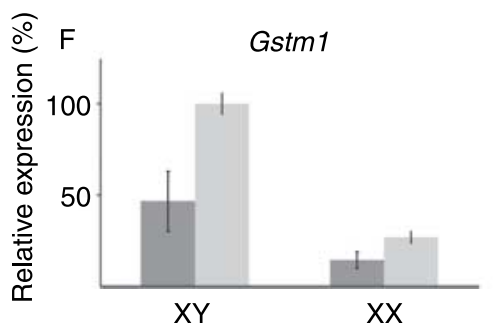

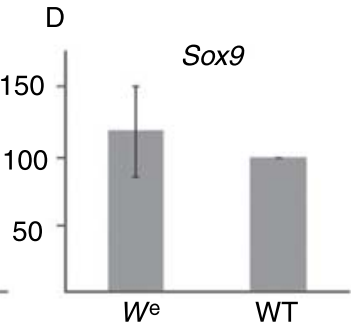

E

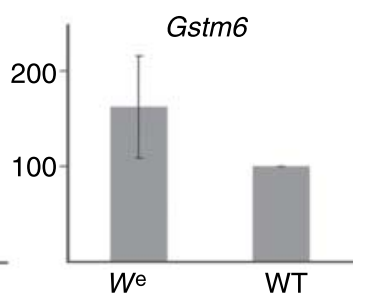

Figure 3 Gstm6 is expressed in Sertoli cells. (A) In situ hybridisation assays using Gstm6 probe 2 showing Gstm6 expression in $13.5 \mathrm{dpc}$ wild-type (left panels) and $W^{e} / W^{e}$ (right panels) gonads showing that Gstm6 expression is maintained at similar levels in gonads lacking germ cells. (B) In situ hybridisation assays using Gstm6 probe 1 showing expression of Gstm6 (left panels), Sertoli cell marker Amh (middle panels) and germ cell marker Pou5f1 (right panels) in $13.5 \mathrm{dpc} \mathrm{XY}$ gonads. Gstm6 is expressed in a pattern similar to Amh but disparate to Pou5f1. Arrow points to presumptive peritubular myoid cells. Scale bars, $100 \mu \mathrm{m}$. (C-E) qRT-PCR assays showing the absence of the germ cell marker $D d \times 4$ in $W^{e} / W^{e}$ gonads (C). Sox9 and Gstm6 expression levels are similar in wild-type and $W^{\mathcal{T}} / W^{\mathcal{e}}$ gonads ( $\mathrm{D}$ and $\mathrm{E}$ ). (F) qRT-PCR assays showing Gstm1 expression levels in WT (dark bars) and $W / W^{e}$ (light bars) XY and XX gonads. (G) qRT-PCR assays showing Gstm2 expression levels in WT (dark bars) and

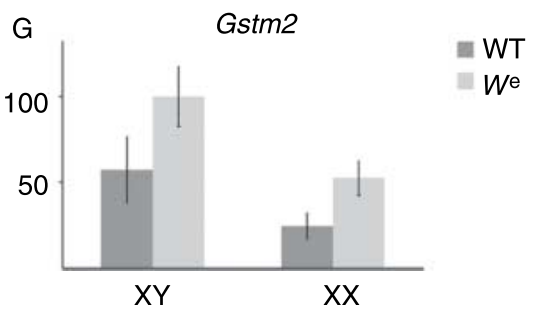

$W^{e} / W^{e}$ (light bars) $\mathrm{XY}$ (left) and XX (right) gonads. The $Y$-axis displays relative fold expression levels compared with $18 S$ rRNA with the gene expression levels in WT gonads set to $100 \%$. Experiments were carried out three times and each experiment consisted of three replicates. The error bars represent the S.E.M. of three experiments. WT, wild type; We, $W^{e} / W^{e}$.

\section{Discussion}

Here, we describe in detail the expression pattern during mouse gonadal development of Gstm6. We found Gstm6 to be expressed at high levels in Sertoli cells of the testis from $12.5 \mathrm{dpc}$ until at least $16.5 \mathrm{dpc}$, but barely or not at all in ovaries. Gstm6 expression was dependent on the expression of Sox9, which controls male sex determination and testis development. Our data suggest that Gstm6 plays a specific role in the development or function of testes. Because GSTs are implicated in the detoxification of endogenous compounds and xenobiotics, Gstm6 expression may provide protection of germ cells against toxicants that may bring about DNA damage or mutation in the germ line. If so, our findings beg the question of why such a role appears to be dispensable in the developing ovaries.

Our experiments also suggested that Gstm6 might be expressed in peritubular myoid cells. This expression may provide an additional level of protection of germ cells in addition to expression in Sertoli cells. Further investigation of this possibility awaits identification of a specific marker gene for peritubular myoid cells (Jeanes et al. 2005).

We observed a temporary downregulation in Gstm6 expression levels in $13.5 \mathrm{dpc}$ XY gonads in qRT-PCR and
WISH assays. This downregulation was observed consistently in sufficient independent trials to exclude error or artefact as an explanation. While the reason for this phenomenon is not understood, it may reflect a

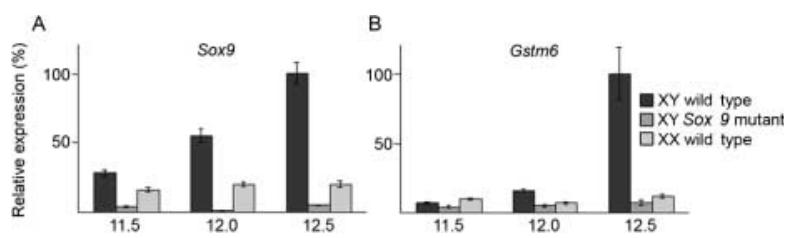

Figure 4 Gstm6 expression is dependent on Sox9. (A) Sox9 expression levels are high in genital ridges and gonads of wild-type XY mice and increase over time, whereas they remain low in genital ridges and gonads of wild-type XX mice. In Sox9, conditional homozygous mutant $\mathrm{XY}$ gonads Sox9 expression levels are strongly reduced when compared with wild-type XY gonads. (B) Gstm6 expression levels are higher in XY genital ridges and gonads and they peak at $12.5 \mathrm{dpc}$, whereas those in genital ridges and gonads of wild-type XX littermates remain low. In Sox9, conditional homozygous mutant XY gonads Gstm6 expression levels are strongly reduced when compared with wild-type $X Y$ gonads, but similar to expression levels found in wild-type XX gonads. The $Y$-axis displays relative fold expression levels compared with $18 S$ rRNA with the maximum expression level of each experiment set to $100 \%$. Each experiment consisted of three replicates. The error bars represent minimal and maximal ranges of gene expression in three replicates. 
period of relatively little metabolic activity following the dramatic morphogenetic events of testicular cord formation. However, from $13.5 \mathrm{dpc}$ onwards, a marked increase in Gstm6 expression levels was observed, perhaps concomitant with the increase in the number of Gstm6-producing Sertoli cells in this period.

We found that that in addition to Gstm6, Gstm1 and Gstm2 also are expressed at higher levels in the somatic cells of XY gonads compared with XX gonads (present study and Beverdam \& Koopman 2006), suggesting that Gstm genes may have redundant functions during gonadogenesis. Further experiments will be required to investigate whether Gstm6 and possibly other Gstm members are indeed involved in detoxification during gonadogenesis or whether they have other, yet unidentified roles in the developing gonads.

\section{Materials and Methods}

\section{Animals}

Protocols and use of animals were approved by the Animal Welfare Unit of the University of Queensland and by the Animal Ethics Committee of Monash University, Australia. Embryos were collected from timed matings of the CD1 outbred strain, the $c-K_{i t}{ }^{W e+/-}$ inbred strain (Buehr et al. 1993) and a transgenic mouse line, maintained on a Swiss outbred background, constitutively expressing EGFP from the X-chromosome (gift of A Nagy; Hadjantonakis et al. 1998) and $0.5 \mathrm{dpc}$ was defined as noon on the day of discovery of the copulation plug. Mouse embryos were staged according to limb bud morphology (Theiler 1989). Embryonic sex was established by assessing GFP fluorescence (for 10.5 and $11.5 \mathrm{dpc}$ samples used for qRT-PCR analyses), by PCR on genomic DNA using Zfy forward primer: 5'-CCTATTGCATGGACTGCAGCTTATG-3' and Zfy reverse primer: 5'-GACTAGACATGTCTTAACATCTGTCC-3' (for other 10.5 and $11.5 \mathrm{dpc}$ samples) or by assessing gonad morphology (for samples $12.5 \mathrm{dpc}$ and older). Ck19:Cre/+; Sox $9^{\text {floxflox }}$ embryos on a 129P2/OlaHsd $\times$ C57BL/6 mixed background were generated, sexed and genotyped as described previously (Daneau et al. 2002, Barrionuevo et al. 2006).

\section{qRT-PCR assays}

Of 10.5 and $11.5 \mathrm{dpc}$ genital ridges and adult and 12.5 up to $16.5 \mathrm{dpc}$ embryonic gonads with mesonephroi attached were pooled according to stage and sex. Total RNA was extracted from dissected embryonic and adult mouse tissues according to the manufacturer's instructions and including the optional genomic DNA degradation I step (Qiagen). Total RNA was reverse transcribed using random hexamer primers (Promega) and Superscript III (Invitrogen) using standard methodologies.

qRT-PCR primers were designed with $\mathrm{Tm}$ of close to $60^{\circ} \mathrm{C}$ to generate $60-145 \mathrm{bp}$ amplicons spanning introns. Target amplification efficiency of all primers was approximately equal. Gstm6-F: 5'-ATGGGCATGCTTTGCTACA-3', Gstm6-R:
5'-GGAACTCCGAGTAGAGTTTCAGC-3', Gstm 1-F: 5'-GCAG CTCATCATGCTCTGTT-3', Gstm 1-R: $5^{\prime}$-TTTTCTCAGGGATGG TCTTCA-3', Gstm2-F: 5'-AGTTGGCCATGGTTTGCTAC-3', Gstm2-R: 5'-AGCTTCATCTTCTCAGGGAGAC-3', Sox9-F: 5'-AGTACCCGCATCTGCACAAC-3', Sox9-R: 5'-TACTTGTAATCGGGGTGGTCT-3', Ddx4-F: $5^{\prime}$-AAGCAGAGGGTTTTCCAAGC-3', Ddx4-R: 5'-GCCTGATGCTTCTGAATCG-3', 185 F: 5'-GATCCATTGGAGGGCAAGTCT-3' and 18S-R: 5'-CCAAGATCCAACTACGAGCTTTTT-3'.

qRT-PCR assays were carried out in triplicate consisting of three replicates each using SYBR green PCR master mix, $3.5 \mu \mathrm{M}$ primer pairs using an ABI Prism 7000 Sequence Detection System following the manufacturer's instructions. Target cDNA levels were analysed by the comparative cycle time $\left(C_{\mathrm{t}}\right)$ method of qRT-PCR and the values were normalised to $18 S$ rRNA expression levels.

\section{In situ hybridisation}

Embryos and embryonic gonads were dissected and fixed in $4 \%$ paraformaldehyde at $4{ }^{\circ} \mathrm{C}$ overnight and washed with PBS. For SISH, embryos were mounted in paraffin wax and sectioned at $7 \mu \mathrm{m}$ using a microtome. SISH was carried out as described by Svingen et al. (2007) and WISH as described by Hargrave et al. (2006).

Gstm6 in situ hybridisation probes were generated by PCR using cDNA from $12.5 \mathrm{dpc}$ mouse gonadal tissue. Gstm6 probe 1 was generated using forward primer 5'-CATGCATGGAGTGCCGGTGT- $3^{\prime}$ and reverse primer $5^{\prime}$-CACACAAGACTGGGCCTTCC-3'. Gstm6 probe 2 was generated using forward primer 5'-ACAAGGTCACCCAGAGCAATGC-3' and reverse primer $5^{\prime}$-TGGCTTCCGTTTCTCAAAGTCAG-3'. PCR fragments were cloned into the pGEM-T Easy vector (Promega), according to the manufacturer's instructions and checked by sequencing. The specificity of each probe was checked by BLAST searching the probe sequence using the NCBI database. Templates for probes for Amh and Pou5f1 were made as described previously (Munsterberg \& Lovell-Badge 1991, Schepers et al. 2003). Gstm6 probe 1 was used for SISH assays, but performed poorly in whole-gonad in situ hybridisation assays where Gstm6 probe 2 was used.

\section{Declaration of interest}

The authors declare that there is no conflict of interest that would prejudice the impartiality of this scientific work.

\section{Funding}

This work was supported by the National Health and Medical Research Council of Australia and the Australian Research Council.

\section{References}

Albrecht KH \& Eicher EM 2001 Evidence that Sry is expressed in pre-Sertoli cells and Sertoli and granulosa cells have a common precursor. Developmental Biology 240 92-107. 
Barrionuevo F, Bagheri-Fam S, Klattig J, Kist R, Taketo MM, Englert C \& Scherer G 2006 Homozygous inactivation of Sox9 causes complete XY sex reversal in mice. Biology of Reproduction 74 195-201.

Beverdam A \& Koopman P 2006 Expression profiling of purified mouse gonadal somatic cells during the critical time window of sex determination reveals novel candidate genes for human sexual dysgenesis syndromes. Human Molecular Genetics 15 417-431.

Bouma GJ, Affourtit JP, Bult CJ \& Eicher EM 2007 Transcriptional profile of mouse pre-granulosa and Sertoli cells isolated from early-differentiated fetal gonads. Gene Expression Patterns 7 113-123.

Buehr M, McLaren A, Bartley A \& Darling S 1993 Proliferation and migration of primordial germ cells in We/We mouse embryos. Developmental Dynamics 198 182-189.

Clement TM, Anway MD, Uzumcu M \& Skinner MK 2007 Regulation of the gonadal transcriptome during sex determination and testis morphogenesis: comparative candidate genes. Reproduction 134 455-472.

Cory AT, Boyer A, Pilon N, Lussier JG \& Silversides DW 2007 Presumptive pre-Sertoli cells express genes involved in cell proliferation and cell signalling during a critical window in early testis differentiation. Molecular Reproduction and Development 74 1491-1504.

Daneau I, Pilon N, Boyer A, Behdjani R, Overbeek PA, Viger R, Lussier J \& Silversides DW 2002 The porcine SRY promoter is transactivated within a male genital ridge environment. Genesis 33 170-180.

Douglas KT 1987 Mechanism of action of glutathione-dependent enzymes. Advances in Enzymology and Related Areas of Molecular Biology $\mathbf{5 9}$ 103-167.

Hadjantonakis AK, Gertsenstein M, Ikawa M, Okabe M \& Nagy A 1998 Generating green fluorescent mice by germline transmission of green fluorescent ES cells. Mechanisms of Development 76 79-90.

Hargrave M, Bowles J \& Koopman P 2006 In situ hybridization of wholemount embryos. Methods in Molecular Biology 326 103-113.

Hayes JD \& Pulford DJ 1995 The glutathione S-transferase supergene family: regulation of GST and the contribution of the isoenzymes to cancer chemoprotection and drug resistance. Critical Reviews in Biochemistry and Molecular Biology 30 445-600.

Hayes JD, Flanagan JU \& Jowsey IR 2005 Glutathione transferases. Annual Review of Pharmacology and Toxicology 45 51-88.

Jeanes A, Wilhelm D, Wilson MJ, Bowles J, McClive PJ, Sinclair AH \& Koopman P 2005 Evaluation of candidate markers for the peritubular myoid cell lineage in the developing mouse testis. Reproduction 130 509-516.

Kent J, Wheatley SC, Andrews JE, Sinclair AH \& Koopman P 1996 A malespecific role for SOX9 in vertebrate sex determination. Development 122 2813-2822.

Koopman P, Munsterberg A, Capel B, Vivian N \& Lovell-Badge R 1990 Expression of a candidate sex-determining gene during mouse testis differentiation. Nature 348 450-452.

Koopman P, Gubbay J, Vivian N, Goodfellow P \& Lovell-Badge R 1991 Male development of chromosomally female mice transgenic for Sry. Nature 351 117-121.
Munsterberg A \& Lovell-Badge R 1991 Expression of the mouse antimullerian hormone gene suggests a role in both male and female sexual differentiation. Development 113 613-624.

Nef S, Schaad O, Stallings NR, Cederroth CR, Pitetti JL, Schaer G, Malki S, Dubois-Dauphin M, Boizet-Bonhoure B, Descombes P et al. 2005 Gene expression during sex determination reveals a robust female genetic program at the onset of ovarian development. Developmental Biology 287 361-377.

De Santa Barbara P, Bonneaud N, Boizet B, Desclozeaux M, Moniot B, Sudbeck P, Scherer G, Poulat F \& Berta P 1998 Direct interaction of SRYrelated protein SOX9 and steroidogenic factor 1 regulates transcription of the human anti-Mullerian hormone gene. Molecular and Cellular Biology 18 6653-6665.

Schepers G, Wilson M, Wilhelm D \& Koopman P 2003 SOX8 is expressed during testis differentiation in mice and synergizes with SF1 to activate the Amh promoter in vitro. Journal of Biological Chemistry 278 28101-28108.

Shen WH, Moore CC, Ikeda Y, Parker KL \& Ingraham HA 1994 Nuclear receptor steroidogenic factor 1 regulates the mullerian inhibiting substance gene: a link to the sex determination cascade. Cell 77 651-661.

Small CL, Shima JE, Uzumcu M, Skinner MK \& Griswold MD 2005 Profiling gene expression during the differentiation and development of the murine embryonic gonad. Biology of Reproduction 72 492-501.

Svingen T, Beverdam A, Verma P, Wilhelm D \& Koopman P 2007 Aard is specifically up-regulated in Sertoli cells during mouse testis differentiation. International Journal of Developmental Biology 51 255-258.

Theiler K 1989 The House Mouse: Atlas of Embryonic Development. New York: Springer-Verlag.

Toyooka Y, Tsunekawa N, Takahashi Y, Matsui Y, Satoh M \& Noce T 2000 Expression and intracellular localization of mouse Vasa-homologue protein during germ cell development. Mechanisms of Development 93 139-149.

Wilhelm D, Hiramatsu R, Mizusaki H, Widjaja L, Combes AN, Kanai Y \& Koopman P 2007 SOX9 regulates prostaglandin D synthase gene transcription in vivo to ensure testis development. Journal of Biological Chemistry 282 10553-10560.

Wilson MJ, Jeyasuria P, Parker KL \& Koopman P 2005 The transcription factors steroidogenic factor- 1 and SOX9 regulate expression of Vanin-1 during mouse testis development. Journal of Biological Chemistry $\mathbf{2 8 0}$ 5917-5923.

Received 8 August 2008

First decision 11 September 2008

Revised manuscript received 8 December 2008

Accepted 19 December 2008 